\title{
ON RICCI SYMMETRIC GENERALIZED QUASI EINSTEIN SPACETIMES
}

\author{
SINEM GÜLER AND SEZGIN ALTAY DEMIRBAĞ
}

Received 30 November, 2014

\begin{abstract}
The object of the present paper is to prove the existence of a generalized quasi Einstein spacetime, briefly $G(Q E)_{4}$, by constructing a non-trivial Lorentzian metric and to study such spacetime. First, we prove that every $W_{2}$-Ricci pseudosymmetric $G(Q E)_{4}$ is an $N(k)$-quasi Einstein spacetime which can be considered as a model of perfect fluid, in general relativity. Then, we consider Ricci symmetric $G(Q E)_{4}$ and we prove that in such spacetime satisfying Einstein's field equations, the energy density and the isotropic pressure are constants. As a consequence of this result, the expansion scalar and the acceleration vector vanish and also the possible local cosmological structures of this spacetime obeying Einstein's field equations are of Petrov I, D or O.
\end{abstract}

2010 Mathematics Subject Classification: 53C15; 53C25; 53B15; 53B20

Keywords: generalized quasi Einstein spacetime, Ricci symmetric spacetime, Codazzi type tensor, Einstein's field equation, energy momentum tensor

\section{INTRODUCTION}

The notion of quasi-Einstein manifold was introduced by Chaki and Maity [3]. A non-flat $n$-dimensional Riemannian manifold $\left(M_{n}, g\right),(n \geqslant 3)$ is said to be quasiEinstein manifold if its Ricci tensor of type $(0,2)$ is not identically zero and satisfies the condition

$$
S(X, Y)=a g(X, Y)+b A(X) A(Y)
$$

where for all vector fields $X$,

$$
g(X, U)=A(X), g(U, U)=1
$$

i.e., $A$ is a non-zero associated 1 -form, metrically equivalent to the unit vector field $U$ and $a, b$ are associated non-zero scalar functions. The vector field $U$ is usually called the generator of the manifold. An n-dimensional manifold of this kind is denoted by $(Q E)_{n}$. Obviously, if $b=0$, then this manifold reduces to an Einstein manifold. From the above definition, it follows that every Einstein manifold is quasiEinstein. In particular, every Ricci-flat manifold (e.g. Schwarzschild spacetime) is quasi-Einstein. 
Quasi Einstein manifolds arose during the study of exact solutions of Einstein's field equations as well as during considerations of quasi-umbilical hypersurfaces of semi-Euclidean spaces. For instance, the Robertson-Walker spacetimes are quasi Einstein manifolds. Also, quasi Einstein manifolds can be taken as a model of perfect fluid spacetime in general relativity, [6]. Thus quasi Einstein manifolds are very important in general relativity and cosmology. These manifolds have been studied by several authors, such as M.C. Chaki [3], U.C. De and G. C. Ghosh [6] and S. Guha [8]. Also, A.A. Shaikh, Y.H. Kim and S.K. Hui [17] have been studied on Lorentzian quasi Einstein spacetimes. Many authors have been generalized the notion of quasi Einstein manifolds, in different ways such as nearly quasi Einstein manifolds [5], generalized quasi Einstein manifolds [2], $N(k)$-quasi Einstein manifolds [4,20] and so on.

In 2001, Chaki [2] introduced the notion of generalized quasi Einstein manifold. An n-dimensional $(n \geqslant 3)$ Riemannian manifold $\left(M_{n}, g\right)$ is said to be generalized quasi Einstein manifold if its Ricci tensor of type $(0,2)$ is not identically zero and satisfies the condition

$$
S(X, Y)=a g(X, Y)+b A(X) A(Y)+c[A(X) B(Y)+A(Y) B(X)]
$$

where $a, b, c$ are real valued, non-zero scalar functions of which $b \neq 0, c \neq 0, A$ and $B$ are non-zero 1 -forms such that

$$
g(X, U)=A(X), g(X, V)=B(X), g(U, V)=0
$$

for all $X, Y \in \chi(M)$. Also, $U$ and $V$ are two unit vector fields perpendicular to each other. Similarly, $a, b$ and $c$ are called associated scalars, $A$ and $B$ are called associated 1-forms and $U$ and $V$ are generators of the manifold. Such an $n$-dimensional manifold has been denoted by $G(Q E)_{n}$. If $c=0$, then (1.3) takes the form (1.1) and so the manifold reduces to a $(Q E)_{n}$. Thus a quasi Einstein manifold is a special case of a generalized quasi Einstein manifold. If $b=c=0$, then the manifold becomes an Einstein manifold. Hence, in a $G(Q E)_{n}$, we may assume that the associated scalar functions $a$ and $b$ are nowhere zero, but $c$ can be any smooth function on the manifold, even constant zero.

Let $\left\{e_{i}: i=1,2, \cdots, n\right\}$ be an orthonormal frame field at any point of the manifold. Then, setting $X=Y=e_{i}$ in (1.3) and taking summation over $i$; $(1 \leq i \leq n)$, we obtain

$$
r=a n+b
$$

where $r$ is the curvature function of the manifold.

Let $R$ denote the Riemannian curvature tensor of $M$. The k-nullity distribution $N(k)$ [19] of a Riemannian manifold $M$ is defined by

$$
\begin{aligned}
& N(k): p \rightarrow N_{p}(k)= \\
& \left\{Z \in T_{p}(M): R(X, Y) Z=k[g(Y, Z) X-g(X, Z) Y] ; \forall X, Y \in T_{p}(M)\right\}
\end{aligned}
$$


where $k$ is some smooth function. In a quasi Einstein manifold $M$, if the generator $U$ belongs to some k-nullity distribution $N(k)$, then $M$ said to be $N(k)$-quasi Einstein manifold [20]. Özgür and Triphati [13] proved that in an $n$-dimensional $N(k)$-quasi Einstein manifold, $k=\frac{a+b}{n-1}$.

A spacetime is a time oriented, 4-dimensional manifold $\left(M^{4}, g\right)$ with Lorentz metric whose signature $(+,+,+,-)$. A Lorentzian 4-dimensional manifold is said to be generalized quasi Einstein spacetime with generator $U$ as the unit timelike vector field if its Ricci tensor of type $(0,2)$ is not identically zero and satisfies the equation (1.3), where again $a, b, c$ are real valued, non-zero scalar functions of which $b \neq 0$, $c \neq 0, A$ and $B$ are non-zero 1-forms such that $V$ being the heat flux vector field orthogonal to the velocity vector field $U$. That is, for all $X$, we have

$$
g(X, U)=A(X), g(X, V)=B(X), g(U, U)=-1, g(V, V)=1, g(U, V)=0
$$

The energy momentum tensor for a perfect fluid spacetime [12] is given by

$$
T(X, Y)=(\sigma+p) A(X) A(Y)+p g(X, Y)
$$

together with $g(X, U)=A(X), A(U)=-1$, for all $X, Y$. Here, $\sigma$ and $p$ are respectively the energy density and isotropic pressure and $U$ is the unit timelike velocity vector field. For a fluid matter distribution, the energy momentum tensor is given by Ellis [7] and Landau and Lifschitz [11] as

$$
T(X, Y)=(\sigma+p) A(X) A(Y)+p g(X, Y)+A(X) B(Y)+A(Y) B(X)
$$

for all $X, Y \in T M$ where $g(X, U)=A(X), A(U)=-1, g(X, V)=B(V), B(V)>$ $0, g(U, V)=0, \sigma$ and $p$ are respectively the energy density and isotropic pressure, $U$ is the velocity vector field orthogonal to the heat conduction vector field $V$.

Ricci tensor controls the geometry of spacetime whereas energy momentum tensor $T$ signifies the physical aspects of spacetime and in general relativity they are related by Einstein's field equations [12]

$$
S(X, Y)-\frac{r}{2} g(X, Y)+\lambda g(X, Y)=k T(X, Y)
$$

for all vector fields $X, Y$ where $S$ is the Ricci tensor of type $(0,2), r$ is the scalar curvature, $\lambda$ is the cosmological constant and $k$ is the gravitational constant. Einstein's field equation implies that the energy momentum tensor is a symmetric $(0,2)$ tensor with divergence zero [12].

This paper is organized as follows:

After introduction, in Section 2, we prove the existence of a generalized quasi Einstein spacetime, briefly $G(Q E)_{4}$, by constructing a non-trivial Lorentzian metric. In Section 3, we prove that every $W_{2}$-Ricci pseudosymmetric $G(Q E)_{4}$ is an $N\left(\frac{a-b}{3}\right)$ quasi Einstein spacetime which can be considered as a model of perfect fluid, in general relativity. In Section 4, a Ricci symmetric $G(Q E)_{4}$ has been considered and a detailed study on this spacetime has been made. We prove that in such spacetime 
satisfying Einstein's field equations, the energy density and the isotropic pressure are constants. Moreover, the expansion scalar and the acceleration vector vanish, also the possible local cosmological structures of this spacetime obeying the Einstein's field equation are of Petrov I, D or O.

\section{EXISTENCE OF A GENERALIZED QUASI EINSTEIN SPACETIME}

The physical motivation for studying various types of spacetime models in cosmology is to obtain the information of different phases in the evolution of the universe, which may be classified into three phases, namely, the initial phase, the intermediate phase and the final phase. The initial phase is just after the Big Bang when the effects of both viscosity and heat flux were quite pronounced. The intermediate phase is that when the effect of viscosity was no longer significant but the heat flux was still not negligible. The final phase, which extends to the present state of the universe when both the effects of viscosity and heat flux have become negligible and the matter content of the universe may be assumed to be perfect fluid. The study of $(Q E)_{4}$ is important because such spacetime represents the third phase in the evolution of the universe. In addition to this, the importance of $G(Q E)_{4}$ lies in the fact that such a 4dimensional semi-Riemannian manifold is related to the study of general relativistic fluid spacetime admitting heat flux represents the intermediate phase in the evolution of the universe, [16]. Thus, in this section, we prove the existence of a generalized quasi Einstein spacetime with non-zero and non-constant scalar curvature by constructing a new Lorentzian metric.

We consider a Lorentzian metric $g$ on the 4-dimensional real number space $\mathbb{R}^{4}$ by

$$
d s^{2}=g_{i j} d x^{i} d x^{j}=\left(x^{4}\right)^{2}\left(d x^{1}\right)^{2}+\left(e^{2 x^{4}}\right)\left[\left(d x^{2}\right)^{2}+\left(d x^{3}\right)^{2}\right]-\left(d x^{4}\right)^{2}
$$

where $\frac{1}{2}<x^{4}<1$ and $x^{1}, x^{2}, x^{3}, x^{4}$ are the standard coordinates of $\mathbb{R}^{4}$. Then the only non vanishing components of the Christoffel symbols, the curvature tensor and the Ricci tensor are obtained as follows:

$$
\begin{gathered}
\Gamma_{14}^{1}=\frac{1}{\left(x^{4}\right)}, \quad \Gamma_{11}^{4}=\left(x^{4}\right), \quad \Gamma_{24}^{2}=\Gamma_{34}^{3}=1, \quad \Gamma_{22}^{4}=\Gamma_{33}^{4}=e^{2\left(x^{4}\right)} \\
R_{1221}=R_{1331}=-e^{2\left(x^{4}\right)}\left(x^{4}\right), \\
R_{2332}=-e^{4\left(x^{4}\right)}, \quad R_{2442}=R_{3443}=e^{2\left(x^{4}\right)} \\
R_{11}=-2\left(x^{4}\right), \quad R_{22}=R_{33}=-e^{2\left(x^{4}\right)} \frac{\left[1+2\left(x^{4}\right)\right]}{\left(x^{4}\right)}, R_{44}=2
\end{gathered}
$$

and the components which can be obtained from these by symmetry properties. Also it can be shown that the scalar curvature is given by

$$
r=-2\left[\frac{2}{\left(x^{4}\right)}+3\right]
$$

which is non-zero and non-constant. 
In terms of local coordinate system, let us define associated scalar functions as

$$
a=-2-\frac{1}{\left(x^{4}\right)}, \quad b=-2, \quad c=\frac{\sqrt{2\left(x^{4}\right)-1}}{\left(x^{4}\right)}
$$

and the 1-forms

$$
A_{i}(x)= \begin{cases}\left(x^{4}\right) \sqrt{\frac{2\left(x^{4}\right)-1}{2\left(1-\left(x^{4}\right)\right)}} & \text { if } i=1 \\ 0 & \text { if } i=2,3 \\ \frac{-1}{\sqrt{2\left(1-\left(x^{4}\right)\right)}} & \text { if } i=4\end{cases}
$$

and

$$
B_{i}(x)= \begin{cases}\frac{\left(x^{4}\right)}{\sqrt{2\left(1-\left(x^{4}\right)\right)}} & \text { if } i=1 \\ 0 & \text { if } i=2,3 \\ -\sqrt{\frac{2\left(x^{4}\right)-1}{2\left(1-\left(x^{4}\right)\right)}} & \text { if } i=4\end{cases}
$$

Then, we can show that

(1) $R_{11}=a g_{11}+b A_{1} A_{1}+2 c A_{1} B_{1}$

(2) $R_{22}=a g_{22}+b A_{2} A_{2}+2 c A_{2} B_{2}$

(3) $R_{33}=a g_{33}+b A_{3} A_{3}+2 c A_{3} B_{3}$

(4) $R_{44}=a g_{44}+b A_{4} A_{4}+2 c A_{4} B_{4}$

Since all the cases other than (1)-(4) are trivial, we obtain

$$
R_{i j}=a g_{i j}+b A_{i} A_{j}+c\left(A_{i} B_{j}+A_{j} B_{i}\right), \text { for } i, j=1,2,3,4
$$

Moreover, we find

$$
g^{i j} A_{i} A_{j}=-1, g^{i j} B_{i} B_{j}=1, \text { and } g^{i j} A_{i} B_{j}=0
$$

and so

$$
r=4 a-b=-2\left[\frac{2}{\left(x^{4}\right)}+3\right]
$$

Therefore, we can say that the manifold under consideration is a generalized quasi Einstein spacetime with non-zero and non-constant scalar curvature. Hence we can state that:

Theorem 1. Let $M^{4}=\left\{\left(x^{1}, x^{2}, x^{3}, x^{4}\right) \in \mathbb{R}^{4}: \frac{1}{2}<x^{4}<1\right\}$ be an open subset of $\mathbb{R}^{4}$ endowed with the Lorentzian metric given by

$$
d s^{2}=g_{i j} d x^{i} d x^{j}=\left(x^{4}\right)^{2}\left(d x^{1}\right)^{2}+\left(e^{2 x^{4}}\right)\left[\left(d x^{2}\right)^{2}+\left(d x^{3}\right)^{2}\right]-\left(d x^{4}\right)^{2}
$$

where $x^{1}, x^{2}, x^{3}, x^{4}$ are the standard coordinates of $\mathbb{R}^{4}$. Then $\left(M^{4}, g\right)$ is a generalized quasi Einstein spacetime with non-zero and non-constant scalar curvature $r=-2\left[\frac{2}{\left(x^{4}\right)}+3\right]$. 


\section{SOME PROPERTIES OF GENERALIZED QUASI EINSTEIN SPACETIMES}

In 1970, G. P. Pokhariyal and R. S. Mishra [15] were introduced the new tensor called $W_{2}$-curvature tensor and studied its properties. According to them, an $W_{2}$ curvature tensor on a manifold $\left(M^{n}, g\right),(n>3)$ is defined by

$$
W_{2}(X, Y) Z=R(X, Y) Z-\frac{1}{n-1}[g(Y, Z) Q X-g(X, Z) Q Y]
$$

where $R$ is the curvature tensor and $Q$ denotes the Ricci operator defined by $g(Q X, Y)=S(X, Y)$, for all $X, Y$. Note that for all $X, Y, Z, W \in T M$, the $W_{2}$ curvature tensor satisfies the following symmetry properties:

- $W_{2}(X, Y, Z, W)=-W_{2}(Y, X, Z, W)$

- $W_{2}(X, Y, Z, W) \neq-W_{2}(X, Y, W, Z)$

Let $\left\{e_{i}\right\}$ be an orthonormal basis of the tangent space at each point of the manifold where $1 \leqslant i \leqslant n$. Now, from (3.1), we have

$$
\sum_{i=1}^{n} W_{2}\left(Y, e_{i}, e_{i}, U\right)=0
$$

In this section, a comprehensive study of $W_{2}$-curvature tensor has been made on $G(Q E)_{4}$. Firstly, we consider a generalized quasi Einstein spacetime satisfying the condition $W_{2} \cdot S=L_{S} Q(g, S)$ where $L_{S}$ is a certain function on the set $U_{S}=\{x \in$ $M: S \neq \frac{r}{n} g$ at $\left.x\right\}$ and $Q(g, S)$ is the Tachibana tensor of the metric tensor and the Ricci tensor. Recall that this spacetime is said to be $W_{2}$-Ricci pseudosymmetric $G(Q E)_{4}$. Then for all $X, Y, Z \in \mathfrak{X}\left(M^{4}\right)$;

$$
\begin{aligned}
& S\left(W_{2}(X, Y) Z, W\right)+S\left(Z, W_{2}(X, Y) W\right) \\
& =L_{S}[g(Y, Z) S(X, W)-g(X, Z) S(Y, W)+g(Y, W) S(Z, X)-g(X, W) S(Y, Z)]
\end{aligned}
$$

Combining (1.3) and (3.3), we get

$$
\begin{aligned}
& -\left(\frac{a}{3}+L_{S}\right)[g(Y, Z) S(X, W)-g(X, Z) S(Y, W)+g(Y, W) S(X, Z) \\
& -g(X, W) S(Y, Z)]+b\left[A\left(W_{2}(X, Y) Z\right) A(W)+A(Z) A\left(W_{2}(X, Y) W\right)\right] \\
& +c\left[A\left(W_{2}(X, Y) Z\right) B(W)+A(W) B\left(W_{2}(X, Y) Z\right)+A(Z) B\left(W_{2}(X, Y) W\right)\right. \\
& \left.+A\left(W_{2}(X, Y) W\right) B(Z)\right]=0
\end{aligned}
$$

Putting $Z=U$ and $W=V$ in (3.4), we get

$$
\begin{aligned}
& -\left(\frac{a}{3}+L_{S}\right)[A(Y) S(X, V)-A(X) S(Y, V)+B(Y) S(X, U)-B(X) S(Y, U)] \\
& -b W_{2}(X, Y, V, U)+c\left[W_{2}(X, Y, U, U)-W_{2}(X, Y, V, V)\right]=0
\end{aligned}
$$

In a generalized quasi Einstein spacetime, from (1.7) we have

$$
S(X, U)=(a-b) A(X)-c B(X), \quad S(X, V)=a B(X)+c A(X)
$$


In view of (3.6) and (3.1), (3.5) yields

$$
b R(X, Y, U, V)=b\left(\frac{2 a-b}{3}+L_{S}\right)[A(Y) B(X)-A(X) B(Y)]
$$

Since $b \neq 0$, we obtain

$$
R(X, Y, U, V)=\left(\frac{2 a-b}{3}+L_{S}\right)[A(Y) B(X)-A(X) B(Y)]
$$

Contracting (3.4) over $X$ and $W$, we get

$$
\begin{aligned}
-\left(\frac{a}{3}+L_{S}\right)[r g(Y, Z)-4 S(Y, Z)] & +b W_{2}(U, Y, Z, U) \\
& +c\left[W_{2}(V, Y, Z, U)+W_{2}(U, Y, Z, V)\right]=0
\end{aligned}
$$

Putting $Z=U$ in (3.9), we get

$$
\begin{aligned}
-\left(\frac{a}{3}+L_{S}\right)[r A(Y)-4 S(Y, U)] & +b W_{2}(U, Y, U, U) \\
+ & c\left[W_{2}(V, Y, U, U)-W_{2}(U, Y, U, V)\right]=0
\end{aligned}
$$

In view of (3.6) and (3.1), (3.10) yields

$$
\begin{aligned}
{\left[-3 b\left(\frac{a}{3}+L_{S}\right)+\frac{c^{2}}{3}\right] A(Y) } & \\
+ & {\left[-4 c\left(\frac{a}{3}+L_{S}\right)+\frac{b c}{3}+c\left(\frac{2 a-b}{3}+L_{S}\right)-\frac{a c}{3}\right] B(Y)=0 }
\end{aligned}
$$

Putting $Y=U$ in (3.11), we get:

$$
L_{S}=\frac{-3 a b+c^{2}}{9 b}
$$

Putting $Y=V$ in (3.11), we get:

$$
c\left(a+3 L_{S}\right)=0
$$

Then, $c=0$ or $L_{S}=\frac{-a}{3}$. If $c=0$, then by (3.12), as $b \neq 0$, we get $L_{S}=\frac{-a}{3}$. Otherwise, if $b=0$, then the spacetimes reduces to an Einstein manifold. On the other hand, if $c \neq 0$, then $L_{S}=\frac{-a}{3}$. Then by (3.12), again $c$ must be zero.

Thus, in each case $c=0$ and $L_{S}=\frac{-a}{3}$. That is, the spacetime reduces to a quasi Einstein spacetime and also by (3.8),

$$
R(X, Y) U=\left(\frac{a-b}{3}\right)[A(Y) X-A(X) Y]
$$

which means that the generator vector field $U$ belongs to $\left(\frac{a-b}{3}\right)$-nullity distribution. Therefore, this manifold becomes an $N\left(\frac{a-b}{3}\right)$-quasi Einstein spacetime. Hence, we can state the following theorem: 
Theorem 2. Every $W_{2}$-Ricci pseudosymmetric generalized quasi Einstein spacetime (i.e. satisfying the condition $W_{2} \cdot S=L_{S} Q(g, S)$ for some certain function $L_{S}$ ) is an $N\left(\frac{a-b}{3}\right)$-quasi Einstein spacetime where $L_{S}=\frac{-a}{3}$.

Similarly, if we consider $W_{2}$-Ricci semisymmetric generalized quasi Einstein spacetime (i.e. satisfying the condition $W_{2} \cdot S=0$ ), from above we obtain $c=0$ and $L_{S}=\frac{-a}{3}=0$. Hence, in this case the Ricci tensor can be written as

$$
S(X, Y)=b A(X) A(Y)
$$

Also, contracting (3.15) over $X$ and $Y$, we obtain $r=-b$. Thus, we get

$$
S(X, Y)=-r A(X) A(Y)
$$

where $r$ is the scalar curvature of the spacetime. Such a spacetime is said to be special quasi Einstein spacetime.

Theorem 3. Every $W_{2}$-Ricci semisymmetric generalized quasi Einstein spacetime (i.e. satisfying the condition $W_{2} \cdot S=0$ ) is a special quasi Einstein spacetime.

Combining (3.16) and Einstein's field equations [12]

$$
S(X, Y)-\frac{r}{2} g(X, Y)+\lambda g(X, Y)=k T(X, Y)
$$

we obtain the energy-momentum tensor of this spacetime as follows:

$$
T(X, Y)=\frac{2 \lambda-r}{2 k} g(X, Y)-\frac{r}{k} A(X) A(Y)
$$

which means that this spacetime can be considered as model of perfect fluid spacetime. Hence we can state that the following:

Corollary 1. Every generalized quasi Einstein spacetime satisfying the condition $W_{2} \cdot S=0$ can be considered as model of perfect fluid spacetime, in general relativity.

Now, we consider a fluid spacetime admitting heat flux with the energy momentum tensor of the form (1.9) and satisfying Einstein's field equations with cosmological constant. In this case, the Ricci tensor can be expressed as

$$
\begin{aligned}
S(X, Y)=(k p+ & \left.\frac{r}{2}-\lambda\right) g(X, Y) \\
& +k(\sigma+p) A(X) A(Y)+k[A(X) B(Y)+A(Y) B(X)]
\end{aligned}
$$

for all vector fields $X, Y$ where $r$ is the scalar curvature, $k$ is the gravitational constant, $T$ is the energy momentum tensor of type $(0,2)$ and $\sigma$ and $p$ are the matter density and pressure of the fluid, respectively. Letting

$$
a=\frac{r}{2}+k p-\lambda, \quad b=k(\sigma+p), c=k
$$

we get the following result: 
Theorem 4. A fluid spacetime admitting heat flux with the energy-momentum tensor of the form (1.9) and satisfying Einstein's field equations with cosmological constant is a generalized quasi Einstein spacetime.

Now, we consider quasi Einstein spacetime satisfying Einstein's field equations and we assume that the matter distribution of this spacetime is a fluid with the basic vector field. Then comparing the equations (1.1) and (3.19), we get

$$
\left(a-\frac{r}{2}-k p+\lambda\right) g(X, Y)+[b-k(\sigma+p)] A(X) A(Y)=k[A(X) B(Y)+A(Y) B(X)]
$$

Putting $Y=U$ in (3.21), we get

$$
\left(a-\frac{r}{2}-k p+\lambda-b+k(\sigma+p)\right) A(X)=-k B(X)
$$

Putting $X=U$ in (3.22) and as $B(U)=0$, we get

$$
a-\frac{r}{2}-k p+\lambda-b+k(\sigma+p)=0
$$

In view of (3.23), as $k \neq 0,(3.22)$ yields $B(X)=0$, for all vector field $X$. Hence we obtain:

Theorem 5. In a quasi Einstein spacetime satisfying Einstein's field equations with cosmological constant, if the matter distribution is fluid with the basic vector field of the spacetime, then this spacetime can not admit heat flux.

Remark 1 . This means that such spacetime describes a universe which has already attained thermal equilibrium.

On the other hand, in a generalized quasi Einstein spacetime satisfying Einstein's field equations with cosmological constant, if the matter distribution is a perfect fluid, then comparing the equations (1.3) and (3.19), we get $c=0$, which is a contradiction. Hence we can state the following:

Theorem 6. There exists no generalized quasi Einstein spacetime satisfying Einstein's field equations with cosmological constant whose matter content is perfect fluid.

In the sequel, we shall determine some physical properties of $W_{2}$-flat spacetime satisfying Einstein's field equations with cosmological constant. That is, in this spacetime, the $W_{2}$-curvature tensor vanishes. Then by (3.1), we have

$$
R(X, Y, Z, W)=\frac{1}{3}[g(Y, Z) S(X, W)-g(X, Z) S(Y, W)]
$$

Contracting (3.24) over $X$ and $W$, we get

$$
S(Y, Z)=\frac{r}{4} g(Y, Z)
$$

which gives the scalar curvature $r$ is constant. 
By virtue of (3.25), (3.24) yields

$$
R(X, Y, Z, W)=\frac{r}{12}[g(Y, Z) g(X, W)-g(X, Z) g(Y, W)]
$$

which also shows that $W_{2}$-flat spacetimes are of constant curvature. From (3.26), we have

$$
R(X, Y) Z=\frac{r}{12}[g(Y, Z) X-g(X, Z) Y]
$$

Let $U^{\perp}$ denote the 3-dimensional distribution in an $W_{2}$-flat spacetime orthogonal to $U$. Then for all $X, Y, Z \in U^{\perp}$, the equation (3.27) holds. Moreover, for every $X \in U^{\perp}$, we obtain

$$
R(X, U) U=\frac{r}{12} X
$$

According to H. Karchar [9], a Lorentzian manifold is called infinitesimally spatially isotropic relative to timelike unit vector field $U$ if its curvature tensor $R$ satisfies the relations

$$
R(X, Y) Z=l[g(Y, Z) X-g(X, Z) Y] ; \quad \forall X, Y, Z \in U^{\perp}
$$

and

$$
R(X, U) U=m X ; \forall X \in U^{\perp}
$$

where $l, m$ are real valued function on the manifold. Hence, (3.27) and (3.28) lead to following result:

Theorem 7. Every $W_{2}$-flat spacetime satisfying Einstein's field equations with the velocity vector field $U$ is infinitesimally spatially isotropic relative to timelike unit vector field $U$.

The geometrical symmetries of a spacetime are expressed through the equation

$$
\mathscr{L}_{\xi} A-2 \Omega A=0
$$

where $A$ represents a geometrical or physical quantity, $\mathscr{L}_{\xi}$ denotes the Lie derivative with respect to a vector field $\xi$ and $\Omega$ is a scalar, [10].

One of the most simple and widely used example is the metric inheritance symmetry for which $A=g_{i j}$ in (3.31). That is,

$$
\left(\mathscr{L}_{\xi} g\right)(X, Y)=2 \Omega g(X, Y)
$$

In this case, $\xi$ is the Killing vector field if $\Omega=0$ and is the conformal Killing vector field if $\Omega$ is a scalar function.

Afterwards of this section, the existence of Killing and conformal Killing vector fields has been established. By Einstein's field equations (3.17) and (3.25) we obtain

$$
\left(\lambda-\frac{r}{4}\right) g(X, Y)=k T(X, Y)
$$


Taking the Lie derivative of both sides of (3.33) and remembering that the scalar curvature $r$ is constant, we get

$$
\left(\lambda-\frac{r}{4}\right)\left(\mathscr{L}_{\xi} g\right)(X, Y)=k\left(\mathscr{L}_{\xi} T\right)(X, Y)
$$

Now, if $\xi$ is conformal Killing vector field, from (3.34) and (3.32), we get

$$
2\left(\lambda-\frac{r}{4}\right) \Omega g(X, Y)=k\left(\mathscr{L}_{\xi} T\right)(X, Y)
$$

where $\Omega$ is a scalar function. In a $W_{2}$ flat spacetime satisfying Einstien's field equation with cosmological constant, from (3.33) and (3.35), as $k \neq 0$ we get

$$
\left(\mathscr{L}_{\xi} T\right)(X, Y)=2 \Omega T(X, Y)
$$

It follows from (3.31) that the energy momentum tensor has the symmetry inheritance property.

Conversely, if (3.36) holds, then by (3.33) and (3.34), as $\lambda, k$ and $r$ are constant, (3.32) holds for some scalar function $\Omega$. Thus $\xi$ is the conformal Killing vector field. If we take $\Omega=0$, then from (3.35) and (3.36), a necessary and sufficient condition for $\xi$ to be a Killing vector field is that Lie derivative of the energy momentum tensor with respect to $\xi$ be zero. Hence we can state that:

Theorem 8. An $W_{2}$-flat spacetime satisfying Einstein's field equations with cosmological constant admits:

(1) a conformal Killing vector field if and only if the energy momentum tensor has the symmetry inheritance property.

(2) a Killing vector field $\xi$ if and only if Lie derivative of the energy momentum tensor with respect to $\xi$ vanishes.

\section{RICCI SYMMETRIC GENERALIZED QUASI EINSTEIN SPACETIMES}

In this section, a Ricci symmetric generalized quasi Einstein spacetime has been considered. Then, in this spacetime, the Ricci tensor of $S$ satisfies $\nabla S=0$ where $\nabla$ denotes the Riemannian connection. It follows from (1.3), we have

$$
\begin{aligned}
\left(\nabla_{Z} S\right)(X, Y)= & d a(Z) g(X, Y)+d b(Z) A(X) A(Y)+b\left[\left(\nabla_{Z} A\right)(X) A(Y)\right. \\
& \left.+A(X)\left(\nabla_{Z} A\right)(Y)\right]+d c(Z)[A(X) B(Y)+A(Y) B(X)] \\
& +c\left[\left(\nabla_{Z} A\right)(X) B(Y)+A(X)\left(\nabla_{Z} B\right)(Y)\right. \\
& \left.+\left(\nabla_{Z} A\right)(Y) B(X)+A(Y)\left(\nabla_{Z} B\right)(X)\right]=0
\end{aligned}
$$

Putting $X=Y=U$ in (4.1), we get

$$
-d a(Z)+d b(Z)-2 c\left(\nabla_{Z} B\right)(U)=0
$$

and again putting $X=Y=V$ in (4.1), we get

$$
d a(Z)+2 c\left(\nabla_{Z} A\right)(V)=0
$$


Since the generators $U$ and $V$ are mutually orthogonal, $g(U, V)=0$ and so $Z(g(U, V))=g\left(\nabla_{Z} U, V\right)+g\left(U, \nabla_{Z} V\right)=0$. This yields,

$$
\left(\nabla_{Z} B\right)(U)=-\left(\nabla_{Z} A\right)(V)
$$

Substracting (4.3) from (4.2) and using the last relation, we obtain

$$
d(b-2 a)(Z)=0, \forall Z
$$

which means that $b-2 a$ is a constant.

Moreover, contracting (4.1) over $X$ and $Y$, it follows from (4.4) that we obtain

$$
d(4 a-b)(Z)=0, \forall Z
$$

and so $4 a-b$ is also a constant. From these results, we conclude that the scalar functions $a$ and $b$ are constants. Then, the equation (4.3) yields $c\left(\nabla_{Z} A\right)(V)=0$. Thus, we have either $c=0$ or $\left(\nabla_{Z} A\right)(V)=0$. If $c=0$, then by (4.1), we get

$$
b\left[\left(\nabla_{Z} A\right)(X) A(Y)+A(X)\left(\nabla_{Z} A\right)(Y)\right]=0
$$

In this case, $b$ must be different than zero. Otherwise, $b=c=0$ which is a contradiction. Thus, we get

$$
\left(\nabla_{Z} A\right)(X) A(Y)+A(X)\left(\nabla_{Z} A\right)(Y)=0
$$

Putting $X=U$ in (4.8), we obtain

$$
\left(\nabla_{Z} A\right)(Y)=0, \forall Y, Z
$$

On the other hand, if $c \neq 0$, then we have $\left(\nabla_{Z} A\right)(V)=0$. Using the last relation and putting $X=U, Y=V$ in (4.1), we obtain

$$
d(c)(Z)=0, \forall Z
$$

and so $c$ is constant. Then, putting $X=V$ in (4.1) and using $\left(\nabla_{Z} A\right)(V)=0$, we get $c\left(\nabla_{Z} A\right)(Y)=0$. Since in this case $c \neq 0$, again we obtain (4.9).

Hence, in each case $\left(\nabla_{Z} A\right)(Y)=g\left(\nabla_{Z} U, Y\right)=0$; for all $Y, Z$. That is, $\nabla_{Z} U=0$, for all $Z$ which implies that the generator vector field $U$ is parallel. Moreover, putting $Z=U$ in the last eqution, we get

$$
\nabla_{U} U=0
$$

Thus, the integral curves of $U$ are geodesics. Hence we can state that:

Theorem 9. In a Ricci symmetric generalized quasi Einstein spacetime, generator vector field $U$ is parallel and the integral curves of $U$ are geodesics.

By virtue of the above theorem, the Riemannian curvature tensor satisfies

$$
R(X, Y) U=\nabla_{X} \nabla_{Y} U-\nabla_{Y} \nabla_{X} U-\nabla_{[X, Y]} U=0
$$

for all $X, Y$. Contacting (4.12), in a Ricci symmetric $G(Q E)_{4}$, we obtain

$$
S(X, U)=(a-b) A(X)-c B(X)=0
$$


Putting $X=U$ and $X=V$ in (4.13), we get $a=b$ and $c=0$, respectively. Hence the Ricci tensor can be written as in the following form:

$$
S(X, Y)=a[g(X, Y)+A(X) A(Y)]
$$

This leads to the following theorem:

Theorem 10. Every Ricci symmetric generalized quasi Einstein spacetime is a quasi Einstein spacetime whose associated scalar functions are constant and equal.

Combining Einstein's field equations (3.17) and (4.14), in a Ricci symmetric generalized quasi Einstein spacetime satisfying Einstein's field equations with cosmological constant, the energy momentum tensor can be written as in the following form

$$
T(X, Y)=\frac{2 \lambda-a}{2 k} g(X, Y)+\frac{a}{k} A(X) A(Y)
$$

Thus, this spacetime can be considered as a model of perfect fluid spacetime, in general relativity. Since the velocity vector field $U$ of this spacetime is parallel and the associated scalar $a$ is constant, the energy momentum tensor given by (4.15) is of Codazzi type (even covariantly constant). Hence we can state that:

Theorem 11. In a Ricci symmetric generalized quasi Einstein spacetime satisfying Einstein's field equations with cosmological constant, the energy momentum tensor is of Codazzi type.

From the equations (3.19), (3.20) and Theorem (10), we get

$$
\sigma=\frac{3 a-2 \lambda}{2 k}, p=\frac{2 \lambda-a}{2 k}
$$

Additionally, since $a$ is constant, $\sigma$ and $p$ are constants. Therefore, we can state that:

Theorem 12. In a Ricci symmetric generalized quasi Einstein spacetime satisfying Einstein's field equations with cosmological constant, the energy density and the isotropic pressure are constants.

If we assume $\sigma>0$ and $p>0$, from the equation (4.16), we have

$$
\frac{a}{2}<\lambda<\frac{3 a}{2}
$$

so we obtain:

Corollary 2. In a Ricci symmetric generalized quasi Einstein spacetime satisfying Einstein's field equations with cosmological constant $\lambda$, if the isotropic pressure and energy density are positive, then $\lambda$ satisfies the relation $\frac{a}{2}<\lambda<\frac{3 a}{2}$.

Further, we have the energy and force equations for a perfect fluid [12], as follows:

$$
U \sigma=g(\operatorname{grad} \sigma, U)=-(\sigma+p) \operatorname{div} U
$$


and

$$
\begin{aligned}
(\sigma+p)\left(\nabla_{U} U\right)=-\operatorname{grad}_{\perp} p & =-\operatorname{grad}-g(\operatorname{grad}, U) U \\
& =-\operatorname{grad} p-(U p) U
\end{aligned}
$$

where the spatial pressure gradient $\operatorname{grad}_{\perp} p$ is the component of $\operatorname{gradp}$ orthogonal to $U$. From Theorem (12), in a Ricci symmetric generalized quasi Einstein spacetime satisfying Einstein's field equations, both $\sigma$ and $p$ are constants. Then by (4.18) and (4.19), we obtain

$$
U \sigma=-(\sigma+p) \operatorname{div} U=0 \text { and }(\sigma+p)\left(\nabla_{U} U\right)=0
$$

Since $\sigma+p \neq 0$, we have

$$
\operatorname{div} U=0 \text { and } \nabla_{U} U=0
$$

Remark 2. $\sigma+p=0$ means that the matter contents of the spacetime satisfy the vacuum like equation of state.

It is known that $d i v U$ represents the expansion scalar and $\nabla_{U} U$ represents the acceleration vector. Thus in view of (4.21), both of them vanish. This leads to following result:

Theorem 13. In a Ricci symmetric generalized quasi Einstein spacetime obeying Einstein's field equation with (or without) cosmological constant, the expansion scalar and the acceleration vector vanish.

Shaikh et. al. [18] proved that in a perfect fluid quasi-Einstein spacetime with Codazzi type energy momentum tensor, the fluid has vanishing vorticity and vanishing shear. Combining this argument, Theorem (10) and Theorem (11), we get the following result:

Theorem 14. In a Ricci symmetric generalized quasi Einstein spacetime obeying the Einstein's field equations, the vorticity and shear tensors vanish.

According to Petrov classification [14] a spacetime can be divided into six types denoted by I, II, III, D, N and O. A. Barnes [1] has been proved that if a perfect fluid spacetime is shear-free, vorticity-free and the velocity vector field of the fluid is hypersurface orthogonal and the energy density is constant over a hypersurface orthogonal to the velocity vector field, then the possible local cosmological structure of the spacetime are of Petrov type I, D or O. Thus by using the Theorem (12) and Theorem (14), we can state the following:

Theorem 15. The possible local cosmological structures of a Ricci symmetric generalized quasi Einstein spacetime obeying the Einstein's field equation are of Petrov I, $D$ or $O$. 


\section{ACKNOWLEDGEMENT}

This work which is a part of the first author's doctoral thesis is supported by Istanbul Technical University. The authors express their sincere thanks to the referees and editors for their valuable comments.

\section{REFERENCES}

[1] A. Barnes, "On shear free normal flows of a perfect fluid," Gen. Relativ. Gravit., vol. 4, no. 2, pp. 105-129, 1973, doi: 10.1007/BF00762798.

[2] M. C. Chaki, “On generalized quasi-einstein manifolds,” Publ. Math. Debrecen, vol. 58, pp. 638691, 2001.

[3] M. C. Chaki and M. R. K., "On quasi-einstein manifolds," Publ. Math. Debrecen, vol. 57, pp. 227-306, 2000.

[4] A. De, U. C. De, and A. K. Gazi, "On a class of n(k)- quasi einstein manifolds," Commun. Korean Math. Soc., vol. 26, no. 4, pp. 623-634, 2011, doi: 10.4134/CKMS.2011.26.4.623.

[5] U. C. De and A. K. Gazi, "On nealy quasi einstein manifolds," Novi Sad J. Math., vol. 38, no. 2, pp. 115-121, 2008.

[6] U. C. De and G. C. Ghosh, "On quasi einstein and special quasi einstein manifolds," Proc. of the Int. Conf. of Mathematics and its Applications, pp. 178-191, 2004.

[7] G. F. R. Ellis, General Relativity and Cosmology, R. K. Sachs, Ed., New York-London, 1971.

[8] S. Guha, "On quasi einstein and generalized quasi einstein manifolds," Facta Universitatis, vol. 3, no. 14 , pp. 821-842, 2003.

[9] H. Karchar, "Infinitesimal characterization of friedmann universes," Arch. Math. Basel, vol. 38, pp. 58-64, 1992, doi: 10.1007/BF01304758.

[10] G. H. Katzin, J. Levine, and W. R. Davis, "Curvature collineations: A fundamental symmetry property of the space-times of general relativity defined by the vanishing lie derivative of the riemann curvature tensor," J. Mathematical Phys., vol. 10, pp. 617-629, 1969, doi: 10.1063/1.1664886.

[11] L. D. Landau and E. M. Lifshitz, "Course of theoretical physics," Fluid Mech., vol. 6, p. 505, 1963.

[12] B. O'Neill, Semi-Riemannian Geometry With Applications to Relativity. New York: Academic Press, 1983.

[13] C. Özgür and M. M. Triphati, "On the concircular curvature tensor of an n(k)-einstein manifolds," Math. Pannon., vol. 18, pp. 95-100, 2007.

[14] A. Z. Petrov, Einstein Spaces. Oxford: Pergamon Press, 1949.

[15] G. P. Pokhariyal and R. S. Mishra, "The curvature tensor and their relativistic significance," Yokohama Math. J., vol. 18, pp. 105-108, 1970.

[16] D. Ray, "Gödel-like cosmological solutions," J. Math. Phys., vol. 21, p. 2797, 1980, doi: 10.1063/1.524401.

[17] A. A. Shaikh, Y. H. Kim, and S. K. Hui, "On lorentzian quasi einstein manifolds," J. Korean Math. Soc., vol. 48, no. 4, pp. 669-689, 2011, doi: 10.4134/JKMS.2011.48.4.669.

[18] A. A. Shaikh, D. W. Yoon, and S. K. Hui, "On quasi einstein spacetimes," Tsukuba Journal Math., vol. 33, no. 2, pp. 305-326, 2009.

[19] S. Tanno, "Ricci curvatures of contact riemannian manifolds," Tohoku Math. J., vol. 40, pp. 441448, 1988, doi: 10.2748/tmj/1178227985.

[20] M. M. Triphati and J. S. Kim, “On n(k)-einstein manifolds," Commun. Korean Math. Soc., vol. 22, no. 3, pp. 411-417, 2007. 
Authors' addresses

\section{Sinem Güler}

Istanbul Technical University, Department of Mathematical Engineering, Maslak, 34469, Istanbul, Turkey

E-mail address: singuler@itu.edu.tr

\section{Sezgin Altay Demirbağ}

Istanbul Technical University, Department of Mathematical Engineering, Maslak, 34469, Istanbul, Turkey

E-mail address: saltay@itu.edu.tr 